\title{
Total perfect codes in Cayley graphs
}

\author{
Sanming Zhou \\ School of Mathematics and Statistics \\ The University of Melbourne \\ Parkville, VIC 3010, Australia \\ Email: smzhou@ms.unimelb.edu.au
}

April 10, 2018

\begin{abstract}
A total perfect code in a graph $\Gamma$ is a subset $C$ of $V(\Gamma)$ such that every vertex of $\Gamma$ is adjacent to exactly one vertex in $C$. We give necessary and sufficient conditions for a conjugation-closed subset of a group to be a total perfect code in a Cayley graph of the group. As an application we show that a Cayley graph on an elementary abelian 2-group admits a total perfect code if and only if its degree is a power of 2 . We also obtain necessary conditions for a Cayley graph of a group with connection set closed under conjugation to admit a total perfect code.

Key words: perfect code; total perfect code; efficient dominating set; efficient open dominating set; total perfect dominating set; Cayley graph

AMS Subject Classification (2010): 05C25, 05C69, 94B99
\end{abstract}

\section{Introduction}

Let $\Gamma$ be a graph with vertex set $V(\Gamma)$ and edge set $E(\Gamma)$, and let $e \geq 1$ be an integer. The ball with centre $v \in V(\Gamma)$ and radius $e$ is the set of vertices of $\Gamma$ with distance at most $e$ to $v$ in $\Gamma$. A code in $\Gamma$ is simply a subset of $V(\Gamma)$. A code $C \subseteq V(\Gamma)$ is called a perfect e-code [22] in $\Gamma$ if the balls with centres in $C$ and radius $e$ form a partition of $V(\Gamma)$, that is, every vertex of $\Gamma$ is at distance no more than $e$ to exactly one vertex of $C$. A code $C$ is said to be a total perfect code [13] in $\Gamma$ if every vertex of $\Gamma$ has exactly one neighbour in $C$. In graph theory, the ball around $v$ with radius $e$ is also called the $e$-neighbourhood of $v$ in $\Gamma$, a perfect 1 -code in a graph is called an efficient dominating set [7, 23] or independent perfect dominating set [25], and a total perfect code is called an efficient open dominating set [17]. Similar to perfect codes, total perfect codes in graphs are fascinating objects of study [17. Moreover, they have potential applications in some practical domains, such as placement of Input/Output devices in a supercomputing network so that each element to be processed is at distance at most one to exactly one Input/Output device [2]. It is known [12] that deciding whether a graph has a total perfect code is NP-complete.

The notions above were evolved from the work in [4, which in turn has a root in coding theory. In the classical setting, a $q$-ary code is a subset $C \subseteq S^{n}$, where $S$ is a nonempty finite set (the alphabet) of size $q$ and $S^{n}$ the set of $n$-tuples (words of length $n$ ) from $S$. A code $C$ is a perfect e-code if every word in $S^{n}$ is at distance no more than $e$ to exactly one codeword of $C$, where the (Hamming) distance between two words is the number of positions in which they differ. In the case when $S$ is a finite field $\operatorname{GF}(q)$, any subspace of the linear space $\operatorname{GF}(q)^{n}$ is called a linear code. Such a linear code can be expressed as $\left\{\mathbf{x} \in \operatorname{GF}(q)^{n}: \mathbf{x} M^{T}=0\right\}$, where $\mathbf{x}$ 
is treated as a row vector and $M$ is a matrix over $\mathrm{GF}(q)$ called the parity check matrix of the code. See [19, 40] for surveys on perfect codes and related definitions in the classical setting.

In [4], Biggs showed that the proper setting for the perfect code problem is the class of distance-transitive graphs. In fact, the $q$-ary perfect $e$-codes of length $n$ in the classical setting are precisely the perfect $e$-codes in Hamming graph $H(n, q)$, which is distance-transitive and is defined to have $q$-ary words of length $n$ as vertices and edges joining pairs of words of Hamming distance one. Perfect codes in distance-transitive graphs were studied in, for example, [3, 4, 16, 18, 36, 37, 38. Since the fundamental work of Delsarte [8], a great amount of work on perfect codes in distance-regular graphs and association schemes in general has been produced. Beginning with [22], perfect codes in general graphs have also attracted considerable attention in the community of graph theory; see [21, 26, 27, 30, 35, 41] for example.

Perfect codes in Cayley graphs are especially charming objects of study. In [28] sufficient conditions for Gaussian and Eisenstein-Jacobi graphs to contain perfect $e$-codes were given; such graphs are certain Cayley graphs on quotients of the rings of Gaussian and Eisenstein-Jacobi integers, respectively. These conditions were proved to be necessary in [42] in the more general setting of cyclotomic graphs. In [29] a certain Cayley graph on the integer quaternions rightmodulo a fixed nonzero element was introduced and perfect 1-codes in it were constructed. In [39] it was proved that there is no perfect 1-code in any Cayley graph on $\operatorname{SL}\left(2,2^{f}\right), f>1$ with respect to any connection set closed under conjugation. In [7] a methodology for constructing E-chains of Cayley graphs was given and was used to construct infinite families of E-chains of Cayley graphs on symmetric groups, where an E-chain is a countable family of nested graphs each containing a perfect 1-code. In [9] perfect 1-codes in a Cayley graph with connection set closed under conjugation were studied, yielding necessary conditions in terms of the irreducible characters of the underlying group. In [25] it was proved that a subset $C$ of a group $G$ closed under conjugation (or a normal subset as is called in [25]) is a perfect 1-code in a Cayley graph on $G$ if and only if there exists a covering from the Cayley graph to a complete graph such that $C$ is a fibre of the corresponding covering projection. Perfect 1-codes in circulants (that is, Cayley graphs on cyclic groups) were studied in [31, 33].

Total perfect codes have also attracted considerable attention in recent years. In [2] 'latticelike' total perfect codes in the lattice $\mathbb{Z}^{n}$ were constructed, and the authors of this paper conjectured that these enumerate all possibilities of such codes. In [6] total perfect codes in the lattice $\mathbb{Z}^{2}$ and in the grid graphs on tori were studied. In [13] it was proved that the tensor product of any number of simple graphs has a total perfect code if and only if each factor has a total perfect code. In [5, 20] the grid graphs that have total perfect codes were characterized. In [24, lexicographic, strong, and disjunctive products of graphs admitting total perfect codes were characterized, and a similar result was also obtained for the cartesian product of any graph with the complete graph of two vertices. Total perfect codes are also related to diameter perfect codes, a notion introduced in [1] for distance regular graphs and adapted in [10] for Lee metric over $\mathbb{Z}^{n}$ and $\mathbb{Z}_{q}^{n}$. In fact, when the Manhattan (for $\mathbb{Z}^{n}$ ) or Lee (for $\mathbb{Z}_{q}^{n}$ ) distance is considered, total perfect codes coincide with diameter perfect codes of minimum distance four, in the sense that if $C$ is a diameter perfect code then $C \cup g C$ is a total perfect code.

Inspired by [25] and [9], in this paper we prove a few results on total perfect codes in Cayley graphs. We first give necessary and sufficient conditions for a subset of a finite group closed under conjugation to be a total perfect code in a Cayley graph of the group (see Theorem 3.3), akin to [25, Theorem 2] for perfect 1-codes. As a key component for this result and its proof, we introduce the concept of pseudocovers of graphs (see Definition 2.4). As an application we show that a Cayley graph on an elementary abelian 2-group admits a total perfect code if and only if its degree is a power of 2 (see Theorem 4.1). This extends [15, Theorem 9.2.3] from hypercubes 
to all Cayley graphs on elementary abelian 2-groups, but our proof technique is different from that in [15]. In $\$ 5$ we give two necessary conditions (see Theorems 5.5 and 5.10 for a Cayley graph with connection set a union of conjugacy classes to contain a total perfect code, by using the irreducible characters of the underlying group. These are parallel to [9, Theorems 6-7] and their proofs are accomplished by using a similar approach.

\section{Preliminaries}

All graphs in the paper are undirected without loops and multi-edges, and all groups considered are finite. Group-theoretic notation and terminology used can be found in most textbooks on group theory; see [34] for an introduction to group theory and [11] for the theory of characters of finite groups. We use 1 to denote the identity element of the group under consideration. An involution in a group is an element of order two. We use $\Gamma[X]$ to denote the subgraph of a graph $\Gamma$ induced by a subset $X$ of $V(\Gamma)$, and $\Gamma(v)$ the neighbourhood of a vertex $v \in V(\Gamma)$ in $\Gamma$ (that is, the set of vertices adjacent to $v$ in $\Gamma$ ).

The following observation follows from the definition of a total perfect code.

Lemma 2.1. Let $\Gamma$ be a graph. A subset $C$ of $V(\Gamma)$ is a total perfect code in $\Gamma$ if and only if

(a) $\Gamma[C]$ is a matching; and

(b) $\{\Gamma(v) \backslash C: v \in C\}$ is a partition of $V(\Gamma) \backslash C$.

In particular, any total perfect code in $\Gamma$ must contain an even number of vertices.

A graph $\Sigma$ is called a cover of a graph $\Gamma$ with covering projection $p: \Sigma \rightarrow \Gamma$ if there exists a surjective mapping $p: V(\Sigma) \rightarrow V(\Gamma)$ such that for each $u \in V(\Sigma)$ the restriction of $p$ to $\Sigma(u)$ is a bijection from $\Sigma(u)$ to $\Gamma(p(u))$. We call $\Sigma$ a $k$-fold cover of $\Gamma$ if all fibres $p^{-1}(v), v \in V(\Gamma)$ have size $k$. The following two lemmas are analogies of [25, Lemmas 1-2].

Lemma 2.2. Let $\Gamma$ be a d-regular graph, where $d \geq 1$.

(a) If $C$ is a total perfect code in $\Gamma$, then

$$
|C|=\frac{|V(\Gamma)|}{d} .
$$

In particular, if $\Gamma$ admits a total perfect code, then d divides $|V(\Gamma)|$ and $|V(\Gamma)| / d$ is even. Thus any regular graph with an odd number of vertices does not admit total perfect codes.

(b) If $C_{1}, \ldots, C_{n}$ are pairwise disjoint total perfect codes in $\Gamma$, then the subgraph of $\Gamma$ with vertex set $\cup_{i=1}^{n} C_{i}$ and edges of $\Gamma$ joining distinct such codes is a c-fold cover of $K_{n}$, where $c=|V(\Gamma)| / d$.

Proof (a) By Lemma 2.1, we have $d|C|=|V(\Gamma)|$, yielding $|C|=|V(\Gamma)| / d$. Thus $d$ is a divisor of $|V(\Gamma)|$ and again by Lemma 2.1. $|V(\Gamma)| / d$ must be even.

(b) By (a), all codes $C_{1}, \ldots, C_{n}$ have size $c=|V(\Gamma)| / d$. By the definition of a total perfect code, the edges of $\Gamma$ between distinct $C_{i}$ and $C_{j}$ form a matching of size $c$. The union of these matchings for all pairs $(i, j), i \neq j$, is precisely the subgraph $\Sigma$ of $\Gamma$ with vertex set $\cup_{i=1}^{n} C_{i}$ and edges of $\Gamma$ between distinct codes $C_{i}$. Let $K_{n}$ be the complete graph with vertices $v_{1}, \ldots, v_{n}$. Define $p: \cup_{i=1}^{n} C_{i} \rightarrow V\left(K_{n}\right)$ such that $p(u)=v_{i}$ if and only if $u \in C_{i}$. Then $p$ is a covering projection from $\Sigma$ to $K_{n}$ so that $\Sigma$ is a $c$-fold cover of $K_{n}$. 
Lemma 2.3. Let $p: \Sigma \rightarrow \Gamma$ be a covering projection. If $C$ is a total perfect code in $\Gamma$, then $p^{-1}(C)$ is a total perfect code in $\Sigma$.

Proof Since $C$ is a total perfect code in $\Gamma$, by Lemma 2.1, $\Gamma[C]$ is a matching. Since $p$ is a covering projection, it follows that $\Sigma\left[p^{-1}(C)\right]$ is a matching. Similarly, since every vertex of $V(\Gamma) \backslash C$ is adjacent to exactly one vertex of $C$, every vertex of $V(\Sigma) \backslash p^{-1}(C)$ is adjacent to at least one vertex of $p^{-1}(C)$. If a vertex $u \in V(\Sigma) \backslash p^{-1}(C)$ is adjacent to $v, v^{\prime} \in p^{-1}(C)$ in $\Sigma$, then $p(u) \in V(\Gamma) \backslash C$ is adjacent to $p(v), p\left(v^{\prime}\right) \in C$ in $\Gamma$, which implies $p(v)=p\left(v^{\prime}\right)$ as $C$ is a total perfect code in $\Gamma$. Since $\Sigma$ is a cover of $\Gamma$, we must have $v=v^{\prime}$. Therefore, $p^{-1}(C)$ is a total perfect code in $\Sigma$.

Definition 2.4. A graph $\Sigma$ is called a pseudocover of a graph $\Gamma$ if there exists a surjective mapping $p: V(\Sigma) \rightarrow V(\Gamma)$ such that $\Sigma\left[p^{-1}(v)\right]$ is a matching for every $v \in V(\Gamma)$ and $p$ is a covering projection from $\Sigma^{*}$ to $\Gamma$, where $\Sigma^{*}$ is the graph obtained from $\Sigma$ by deleting the matching in each $\Sigma\left[p^{-1}(v)\right]$. We call $p$ a pseudocovering, written $p: \Sigma \rightarrow \Gamma$, and $p^{-1}(v), v \in V(\Gamma)$ the fibres of $p$.

A pseudocovering $p: \Sigma \rightarrow \Gamma$ is called a $G$-pseudocovering if $G$ is a subgroup of $\operatorname{Aut}(\Sigma)$ and there exists an isomorphism $h: \Gamma \rightarrow \Sigma / \mathcal{P}_{G}$ such that the quotient mapping $\Sigma \rightarrow \Sigma / \mathcal{P}_{G}$ is the composition of $p$ and $h$, where $\mathcal{P}_{G}$ is the partition of $V(\Sigma)$ into $G$-orbits and $\Sigma / \mathcal{P}_{G}$ is the quotient graph of $\Sigma$ with respect to $\mathcal{P}_{G}$.

The following lemma is the counterpart of [25, Theorem 1] for total perfect codes.

Lemma 2.5. Let $\Gamma$ be a regular graph with $E(\Gamma) \neq \emptyset$ and $n$ a positive integer. Then $\Gamma$ is a pseudocover of $K_{n}$ if and only if $V(\Gamma)$ admits a partition $\left\{C_{1}, \ldots, C_{n}\right\}$ such that each $C_{i}$ is a total perfect code in $\Gamma, i=1, \ldots, n$.

Proof The sufficiency follows from Lemma 2.2(b) and Definition 2.4 immediately.

Suppose $\Gamma$ is a pseudocover of $K_{n}$ with a pseudocovering projection $p: V(\Gamma) \rightarrow V\left(K_{n}\right)$. Then for each $v \in V\left(K_{n}\right)$ the fibre $p^{-1}(v)$ is a total perfect code in $\Gamma$, and all such fibres form a partition of $V(\Gamma)$ as required.

\section{Total perfect codes in Cayley graphs}

Given a group $G$ and a subset $S$ of $G$ such that $1 \notin S$ and $S=S^{-1}:=\left\{g^{-1}: g \in S\right\}$, the Cayley graph $\operatorname{Cay}(G, S)$ on $G$ with respect to the connection set $S$ is defined to have vertex set $G$ such that $x, y \in G$ are adjacent if and only if $x y^{-1} \in S$. Obviously, Cay $(G, S)$ is an undirected graph of degree $|S|$ without loops.

Lemma 3.1. Suppose $C \subseteq G$ is a total perfect code in a Cayley graph $\operatorname{Cay}(G, S)$. Then

(a) for every $g \in S, C g$ is a total perfect code in $\operatorname{Cay}(G, S)$;

(b) $\{g C: g \in S\}$ is a partition of $G$;

(c) if $S$ is closed under conjugation, then $\{C g: g \in S\}$ is a partition of $G$.

Proof Denote $\Gamma=\operatorname{Cay}(G, S)$.

(a) It is clear that any automorphism of $\Gamma$ leaves the set of total perfect codes in $\Gamma$ invariant. In particular, since for every $g \in S, \hat{g}: x \mapsto x g, x \in G$ defines an automorphism of $\Gamma, C g$ must be a total perfect code in $\Gamma$. 
(b) Suppose $g_{1} C \cap g_{2} C \neq \emptyset$ for distinct $g_{1}, g_{2} \in S$. Then there exist $x_{1}, x_{2} \in C$ such that $g_{1} x_{1}=g_{2} x_{2}$. Since $g_{1} \neq g_{2}$, we have $x_{1} \neq x_{2}$. Thus $g_{1} x_{1}$ is adjacent to distinct vertices $x_{1}, x_{2}$ in $C$, contradicting the assumption that $C$ is a total perfect code in $\Gamma$. Thus $g_{1} C \cap g_{2} C=\emptyset$ for distinct $g_{1}, g_{2} \in S$. Moreover, since $\Gamma$ is $|S|$-regular, we have $|C||S|=|G|$ by (1) and therefore $\{g C: g \in S\}$ is a partition of $G$.

(c) Similar to (b), it suffices to prove $C g_{1} \cap C g_{2}=\emptyset$ for distinct $g_{1}, g_{2} \in S$. Suppose otherwise, say, $x_{1} g_{1}=x_{2} g_{2}$ for $x_{1}, x_{2} \in C$. Since $g_{1} \neq g_{2}$, we have $x_{1} \neq x_{2}$. Since $S$ is closed under conjugation, we have $h_{1}:=x_{2} g_{2} x_{1}^{-1}=x_{1} g_{1} x_{1}^{-1} \in S$ and $h_{2}:=x_{1} g_{1} x_{2}^{-1}=x_{2} g_{2} x_{2}^{-1} \in S$. Since $h_{1} x_{1}=x_{2} g_{2}=x_{1} g_{1}=h_{2} x_{2}$, by (b) above we have $h_{1}=h_{2}$ and hence $x_{1}=x_{2}$, a contradiction.

The following result follows from Lemmas 2.5 and 3.1 immediately.

Corollary 3.2. Suppose $C \subseteq G$ is a total perfect code in a Cayley graph Cay $(G, S)$. If $g C=C g$ for every $g \in S$, then there exists a pseudocovering $p: \operatorname{Cay}(G, S) \rightarrow K_{|S|}$ such that $g C, g \in S$ are the fibres of $p$. Moreover, if $C$ is a normal subgroup of $G$, then $p$ is a $C$-pseudocovering.

The main results in this section are Theorem 3.3 and Corollary 3.4 below, which are counterparts of [25, Theorem 2] and [25, Corollary 2], respectively. As usual we denote $S^{2}:=\left\{g g^{\prime}\right.$ : $\left.g, g^{\prime} \in S\right\}$ for a subset $S$ of a group.

Theorem 3.3. Suppose $C$ is a subset of a group $G$ closed under conjugation. Then the following are equivalent:

(a) $C$ is a total perfect code in $\operatorname{Cay}(G, S)$;

(b) there exists a pseudocovering $p: \operatorname{Cay}(G, S) \rightarrow K_{|S|}$ such that $g C$ is a fibre of $p$ for at least one element $g \in S$;

(c) C satisfies

$$
|C||S|=|G|, C \cap\left(\left(S^{2} \backslash\{1\}\right) C\right)=\emptyset .
$$

Proof Denote $\Gamma=\operatorname{Cay}(G, S)$.

(a) $\Rightarrow$ (b) This follows from Corollary 3.2 immediately.

(b) $\Rightarrow$ (a) Since $K_{|S|}$ is connected, all fibres of $p$ have the same size. Since one of these fibres is assumed to be $g C$ for some $g \in S$, all fibres of $p$ should have size $|g C|=|C|$. Hence $|C||S|=|G|$. By Lemma 2.5, $g C$ is a total perfect code in $\Gamma$. Since $C$ is closed under conjugation, we have $(g C) g^{-1}=C$. Since $g^{-1} \in S$, by Lemma 3.1(a), $C$ is a total perfect code in $\Gamma$.

(a) $\Rightarrow$ (c) Suppose that $C$ is a total perfect code in $\Gamma$. Since (a) and (b) are equivalent as proved above, we have $|C||S|=|G|$ by the argument in the previous paragraph. It remains to prove that $C \cap\left(\left(S^{2} \backslash\{1\}\right) C\right)=\emptyset$. Suppose otherwise. Then there exist $x_{1}, x_{2} \in C$ and $g_{1}, g_{2} \in S$ with $g_{1} g_{2} \neq 1$ such that $x_{1}=g_{1} g_{2} x_{2}$. Thus $x_{1} \neq x_{2}$ and $g_{1}^{-1} x_{1}=g_{2} x_{2}$. Hence $g_{2} x_{2}$ is adjacent to distinct vertices $x_{1}, x_{2}$ in $C$, contradicting the assumption that $C$ is a total perfect code in $\Gamma$.

(c) $\Rightarrow$ (a) The assumption $C \cap\left(\left(S^{2} \backslash\{1\}\right) C\right)=\emptyset$ implies $g_{1} C \cap g_{2} C=\emptyset$ for distinct $g_{1}, g_{2} \in S$. This together with the assumption $|C||S|=|G|$ implies that $\{g C: g \in S\}$ is a partition of $G$. Thus the neighbourhood of $C$ in $\Gamma$ is given by

$$
\cup_{x \in C} \Gamma(x)=\cup_{x \in C} S x=\cup_{g \in S} g C=G .
$$

In other words, every element of $G \backslash C$ is adjacent to at least one element of $C$ in $\Gamma$. If an element $z \in G \backslash C$ is adjacent to distinct $x_{1}, x_{2} \in C$, then there exist $g_{1}, g_{2} \in S$ such that $z=g_{1} x_{1}=g_{2} x_{2}$. Since $x_{1} \neq x_{2}$, we have $g_{1} \neq g_{2}$ and so $x_{1}=g_{1}^{-1} g_{2} x_{2} \in C \cap\left(\left(S^{2} \backslash\{1\}\right) C\right)$, contradicting the 
assumption $C \cap\left(\left(S^{2} \backslash\{1\}\right) C\right)=\emptyset$. Therefore, every element of $G \backslash C$ is adjacent to a unique element of $C$ in $\Gamma$.

Since $\{g C: g \in S\}$ is a partition of $G$, every element $x \in C$ is of the form $x=g_{1} x_{1}$ for some $g_{1} \in S$ and $x_{1} \in C$, and so $x$ is adjacent to $x_{1}$ in $\Gamma$. If $x$ is also adjacent to some $x_{2} \in C$ with $x_{2} \neq x_{1}$, then $x=g_{2} x_{2}$ for some $g_{2} \in S$. So $g_{1} \neq g_{2}$ and $g_{1} x_{1}=g_{2} x_{2} \in g_{1} C \cap g_{2} C$, which is a contradiction. Therefore, every element of $C$ is adjacent to a unique element of $C$ in $\Gamma$. This together with what we proved in the previous paragraph implies that $C$ is a total perfect code in $\Gamma$.

Corollary 3.4. Let $N$ be a normal subgroup of a group $G$. Then the following are equivalent:

(a) $N$ is a total perfect code in $\operatorname{Cay}(G, S)$;

(b) $\operatorname{Cay}(G, S)$ is an $N$-pseudocovering graph of $K_{|S|}$;

(c) $N$ satisfies

$$
|G: N|=|S|, \quad N \cap S^{2}=\{1\} .
$$

Moreover, if one of these conditions holds, then $|N \cap S|=1$ and the unique element of $N \cap S$ must be an involution.

Proof By Corollary 3.2 and Theorem 3.3 , to prove the equivalence of (a), (b) and (c), it suffices to prove that $N \cap\left(\left(S^{2} \backslash\{1\}\right) N\right)=\emptyset$ if and only if $N \cap S^{2}=\{1\}$.

Suppose $N \cap\left(\left(S^{2} \backslash\{1\}\right) N\right)=\emptyset$. Since $N$ is a subgroup of $G$, we have $1 \in N$ and so $N \cap\left(S^{2} \backslash\{1\}\right)=\emptyset$. In other words, $N \cap S^{2}=\{1\}$.

Now suppose $N \cap\left(\left(S^{2} \backslash\{1\}\right) N\right) \neq \emptyset$. Then there exist $x_{1}, x_{2} \in N$ and $g_{1}, g_{2} \in S$ such that $x_{1}=g_{1} g_{2} x_{2}$ and $g_{1} g_{2} \neq 1$. Since $N$ is a subgroup of $G$, we have $x_{1} x_{2}^{-1}=g_{1} g_{2} \in N \cap S^{2}$ and hence $N \cap S^{2} \neq\{1\}$.

Suppose that one of (a)-(c) holds so that $N$ is a total perfect code in Cay $(G, S)$. Since $1 \in N$ and the set of neighbours of 1 in $N$ is $N \cap S$, we have $|N \cap S|=1$ by the definition of a total perfect code. Let $g$ be the unique element of $N \cap S$. Then $g^{2} \in N \cap S^{2}$ and so $g^{2}=1$ by (c).

Corollary 3.4 imposes strong conditions for a normal subgroup $N$ of $G$ to be a total perfect code in $\operatorname{Cay}(G, S)$. In the same fashion, one can show that for a normal subgroup $N$ of $G$ and an element $g \in S \backslash N, N \cup g N$ is a total perfect code in Cay $(G, S)$ if and only if $|G: N|=2|S|$, $N \cap S^{2}=\{1\}$ and $N \cap S=g N \cap S^{2}=g^{-1} N \cap S^{2}=\emptyset$.

Using additive notation for abelian groups, we write $0, S+S,-S$ and $C+x$ in place of $1, S^{2}, S^{-1}$ and $C x$, respectively. Denote $C-C=\left\{g-g^{\prime}: g, g^{\prime} \in C\right\}$ for any subset $C$ of an abelian group.

Corollary 3.5. Let $G$ be an abelian group. A Cayley graph $\operatorname{Cay}(G, S)$ on $G$ admits a total perfect code if and only if there exists a subset $C$ of $G$ such that $|C||S|=|G|$ and $(C-C) \cap(S+S)=\{0\}$; under these conditions $C$ is a total perfect code in Cay $(G, S)$. Moreover, a subgroup $C$ of $G$ is a total perfect code in $\operatorname{Cay}(G, S)$ if and only if $|C||S|=|G|$ and $C \cap(S+S)=\{0\}$.

Example 3.6. Let Cay $\left(\mathbb{Z}_{n}, S\right)$ be a circulant graph, where $S \subseteq \mathbb{Z}_{n} \backslash\{[0]\}$ satisfies $-S=S$. By Corollary 3.5. Cay $\left(\mathbb{Z}_{n}, S\right)$ admits a total perfect code that is a subgroup of $\mathbb{Z}_{n}$ if and only if $|S|=m$ is a divisor of $n$ and for any $\left[g_{1}\right],\left[g_{2}\right] \in S, g_{1}+g_{2}$ is not a multiple of $m$ unless it is a multiple of $n$. Moreover, in this case $C=\{[k m]: k \in \mathbb{Z}\}$ is a total perfect code in $\operatorname{Cay}\left(\mathbb{Z}_{n}, S\right)$.

As a concrete example, the circulant $\operatorname{Cay}\left(\mathbb{Z}_{18}, S\right)$ where $S=\{[1],[9],[17]\}$ admits $C=$ $\{[0],[3],[6],[9],[12],[15]\}$ as a total perfect code because $|S|$ divides 18 and [0] is the only common element of $C$ and $S+S=\{[0],[2],[8],[10],[16]\}$. 
Similarly, $\operatorname{Cay}\left(\mathbb{Z}_{20}, S\right)$ with $S=\{[1],[2],[10],[18],[19]\}$ admits $C=\{[0],[5],[10],[15]\}$ as a total perfect code.

\section{Total perfect codes in cubelike graphs}

The $n$-dimensional hypercube $Q_{n}$ is the Cayley graph on the elementary abelian 2-group $\mathbb{Z}_{2}^{n}$ with respect to the set of vectors with exactly one nonzero coordinate. In general, any Cayley graph on $\mathbb{Z}_{2}^{n}, n \geq 1$ is said to be cubelike (a notion introduced by L. Lovász according to [32]). The following result is a generalization of [15, Theorem 9.2.3], where the same necessary and sufficient condition was given in the special case of hypercubes.

Theorem 4.1. A connected cubelike graph admits a total perfect code if and only if its degree is a power of 2. Moreover, we give a construction of linear total perfect codes in any cubelike graph with degree a power of 2 .

Proof We identify $\mathbb{Z}_{2}^{n}$ with the additive group of the $n$-dimensional linear space $V(n, 2)$ over $\mathrm{GF}(2)$. The vectors of $V(n, 2)$ are treated as row vectors and the zero vector of $V(n, 2)$ is denoted by $\mathbf{0}_{n}$. Since the operation of $\mathbb{Z}_{2}^{n}$ is addition of vectors, we use $C+\mathbf{x}$ and $S+S$ in place of $C \mathbf{x}$ and $S^{2}$ respectively.

Let $\Gamma=\operatorname{Cay}\left(\mathbb{Z}_{2}^{n}, S\right)$ be a connected cubelike graph with degree $d$. Then $S=\left\{\mathbf{u}_{1}, \ldots, \mathbf{u}_{d}\right\} \subseteq$ $V(n, 2) \backslash\left\{\mathbf{0}_{n}\right\}$ for $d$ distinct vectors $\mathbf{u}_{1}, \ldots, \mathbf{u}_{d} \in V(n, 2) \backslash\left\{\mathbf{0}_{n}\right\}$. Since $\Gamma$ is connected, $S$ must be a generating set of $\mathbb{Z}_{2}^{n}$. In other words, $S$ contains a basis of $V(n, 2)$, and hence $n \leq d$.

Suppose $\Gamma$ admits a total perfect code. By Lemma 2.2, $d$ is a divisor of the order $2^{n}$ of $\Gamma$. Thus $d=2^{t}$ for some $1 \leq t<n \leq 2^{t}$ and the necessity is proved.

To prove the sufficiency we assume that the degree of $\Gamma$ is of the form $d=2^{t}$ for some integer $t$ with $1 \leq t<n \leq 2^{t}$. By Corollary 3.4 , it suffices to prove the existence of a subgroup $C$ of $\mathbb{Z}_{2}^{n}$ with index $\left|\mathbb{Z}_{2}^{n}: C\right|=2^{t}$ such that $C \cap(S+S)=\left\{\mathbf{0}_{n}\right\}$. We achieve this by constructing such a subgroup $C$ explicitly with the help of an appropriate matrix over $\mathrm{GF}(2)$.

Since $1 \leq t<n \leq d=2^{t}$, there exists a $d \times n$ matrix $Q$ of rank $n$ over $\operatorname{GF}(2)$ such that the rows of $Q P$ give all vectors of $V(t, 2)$, where

$$
P=\left[\begin{array}{c}
I_{t} \\
0_{(n-t) \times t}
\end{array}\right]
$$

with $I_{t}$ the identity matrix and $0_{(n-t) \times t}$ the zero-matrix of corresponding dimensions. (In fact, we may add $n-t$ column vectors of dimension $d$ to the $d \times t$ matrix whose rows are the vectors of $V(t, 2)$ such that the resultant matrix $Q$ has rank $n$.) Since $S$ contains a basis of $V(n, 2)$, the matrix $U$ with rows $\mathbf{u}_{1}, \ldots, \mathbf{u}_{d}$ is a $d \times n$ matrix of rank $n$. Since $Q$ and $U$ have the same dimension and rank, there exists a non-singular $n \times n$ matrix $R$ over $\operatorname{GF}(2)$ such that $Q=U R$. The non-singularity of $R$ implies that $M=R P$ is an $n \times t$ matrix with rank $t$. Thus the null space $\left\{\mathbf{x} \in V(n, 2): \mathbf{x} M=\mathbf{0}_{t}\right\}$ of $M$ is an $(n-t)$-dimensional subspace of $V(n, 2)$. Therefore, its additive group $C$ is a subgroup of $\mathbb{Z}_{2}^{n}$ with $\left|\mathbb{Z}_{2}^{n}: C\right|=2^{t}$. On the other hand, since the rows of $U M=U(R P)=Q P$ give all vectors of $V(t, 2)$, the vectors $\mathbf{u}_{i} M, i=1, \ldots, d$, are pairwise distinct. In other words, $\left(\mathbf{u}_{i}+\mathbf{u}_{j}\right) M \neq \mathbf{0}_{t}$ for $i \neq j$, or equivalently $C \cap(S+S)=\left\{\mathbf{0}_{n}\right\}$. Therefore, by Corollary 3.4. $C$ is a total perfect code in $\Gamma$. Obviously, $C$ is a linear code with the transpose of $M$ as its parity check matrix.

The proof above gives an explicit construction of a linear total perfect code $C$ in the cubelike graph Cay $\left(\mathbb{Z}_{2}^{n}, S\right)$. By Lemma 3.1, the cosets $C+\mathbf{u}_{i}, i=1, \ldots, d$, are all total perfect codes in Cay $\left(\mathbb{Z}_{2}^{n}, S\right)$ and they form a partition of the whole space $V(n, 2)$. 
It was observed by an anonymous referee of this paper that not every total perfect code in a cubelike graph with degree a power of 2 is of the form $C+\mathbf{u}_{i}$ above. For example, let $n=2^{t}$, $t \geq 4$, and let $C$ be a non-linear perfect code in $Q_{n-1}$ containing $\mathbf{0}_{n-1}$ (there are many such non-linear perfect codes as seen in [19]). Then $\{0,1\} \times C$ is a total perfect code in $Q_{n}$, but it is not a coset of a linear total perfect code.

Theorem 4.1 implies the following result.

Corollary 4.2. ([15, Theorem 9.2.3]) The hypercube $Q_{d}$ admits a total perfect code if and only if $d=2^{t}$ for some integer $t \geq 1$.

In [15, Section 9.2.1] the sufficiency was proved by showing that the direct sum of $\{0,1\}$ and the well known Hamming code $H_{t}$ (that is, adding 0 or 1 at the beginning of each codeword of $H_{t}$ ) is a total perfect code of size $2^{n-t}$. This code is exactly the code $C$ constructed in the proof of Theorem 4.1 in the special case $Q_{d}=\operatorname{Cay}\left(\mathbb{Z}_{2}^{d}, S\right)$ with $d=2^{t}$, where $S=\left\{\mathbf{e}_{1}, \ldots, \mathbf{e}_{d}\right\}$ is the standard basis of $V(d, 2)$. This is because in this case the columns of the parity check matrix of $C$ (that is, the rows of $M$ ) are all vectors of $V(t, 2)$ whilst the columns of the parity check matrix of $H_{t}$ are all nonzero vectors of $V(t, 2)$. Note that $C+\mathbf{e}_{i}, i=1, \ldots, d$, are also total perfect codes in $Q_{d}$. A related conjecture [15, 9.4.1] asserts that, if $d=2^{t}, t \geq 3$, then every dominating set of $Q_{d}$ with minimum size is a total perfect code.

Example 4.3. By Corollary $4.2, Q_{4}$ admits total perfect codes. Choose

$$
M=\left[\begin{array}{ll}
1 & 0 \\
0 & 1 \\
1 & 1 \\
0 & 0
\end{array}\right] .
$$

Clearly $M$ has rank 2 and its rows are pairwise distinct. The additive group of the null space of $M$ is $C_{0}=\{(0,0,0,0),(1,1,1,0),(0,0,0,1),(1,1,1,1)\}$. By Corollary 4.2 , $C_{0}$ is a total perfect code in $Q_{4}$. Moreover, by Lemma 3.1 , the following are all total perfect codes in $Q_{4}: C_{0}+\mathbf{e}_{1}=$ $\{(1,0,0,0),(0,1,1,0),(1,0,0,1),(0,1,1,1)\} ; C_{0}+\mathbf{e}_{2}=\{(0,1,0,0),(1,0,1,0),(0,1,0,1),(1,0,1,1)\} ;$ $C_{0}+\mathbf{e}_{3}=\{(0,0,1,0),(1,1,0,0),(0,0,1,1),(1,1,0,1)\}$. (Note that $\left.C_{0}+\mathbf{e}_{4}=C_{0}.\right)$ These four codes form a partition of $V(4,2)$; see Figure 1 for an illustration.

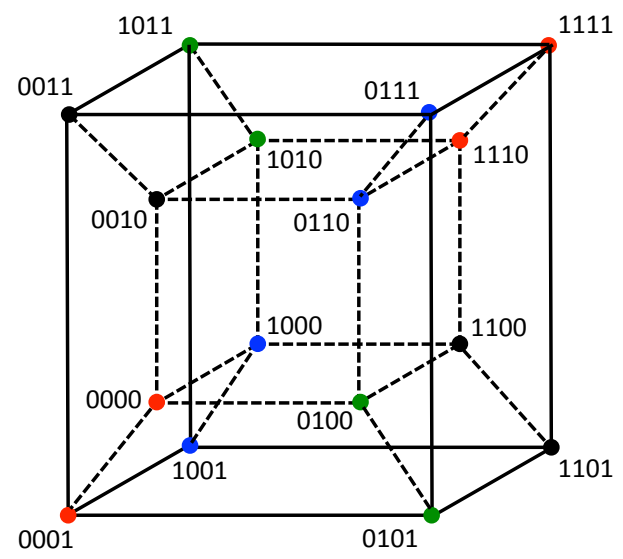

Figure 1: Total perfect codes in $Q_{4}$.

In contrast to $\mathbb{Z}_{2}^{n}$, by Lemma 2.2 (a), for any odd prime $p$, any Cayley graph on $\mathbb{Z}_{p}^{n}$ does not admit any total perfect code. 


\section{$5 \quad$ Necessary conditions}

Interesting necessary conditions for the existence of a perfect 1-code in a directed Cayley graph were given in [9] by using characters of the underlying group. In this section we prove analogous results for total perfect codes in undirected Cayley graphs with connection sets a union of conjugacy classes of the underlying group, by adapting the methodologies in [9]. We begin with a necessary condition for the existence of a total perfect code in a regular (but not necessarily Cayley) graph in terms of equitable partitions, and then apply it to two specific equitable partitions of the above-mentioned Cayley graphs.

\subsection{Total perfect codes and equitable partitions}

In this subsection $\Gamma=(V, E)$ is a $d$-regular graph, where $d \geq 1$, with adjacent matrix

$$
A=\left(a_{u v}\right)_{u, v \in V}
$$

An equitable partition [14, Section 9.3] of $\Gamma$ is a partition $\pi=\left\{V_{1}, \ldots, V_{m}\right\}$ of $V$ such that for every pair $i, j$, the number of neighbours of $u \in V_{i}$ in $V_{j}$ is a constant $b_{i j}$ independent of the choice of $u$. In [9] an equitable partition is called a regular partition.

Denote by $\mathbb{C} V$ the vector space of functions from $V$ to the field of complex numbers $\mathbb{C}$, with addition and scalar multiplication defined in the usual way. As $V$ is finite, we may identify every $f \in \mathbb{C} V$ with the column vector $(f(v))_{v \in V}$. For each $v \in V$, define $\delta_{v} \in \mathbb{C} V$ such that $\delta_{v}(u)=1$ if $u=v$ and $\delta_{v}(u)=0$ if $u \neq v$. Then every $f \in \mathbb{C} V$ can be written as $f=\sum_{v \in V} f(v) \delta_{v}$. Define the 'adjacency' linear mapping [9] by

$$
\varphi: \mathbb{C} V \rightarrow \mathbb{C} V, \quad \delta_{v} \mapsto \varphi\left(\delta_{v}\right):=\sum_{u \in \Gamma(v)} \delta_{u}=\sum_{u \in V} a_{u v} \delta_{u}
$$

It is straightforward to verify [9] that the matrix of $\varphi$ with respect to the 'standard' basis $\left\{\delta_{v}: v \in V\right\}$ of $\mathbb{C} V$ is exactly the adjacency matrix $A$ of $\Gamma$. Alternatively, if $\mathbb{C} V$ is viewed as a vector space of column vectors, then $\varphi$ maps $f \in \mathbb{C} V$ to $A f$.

Define $\delta_{U}=\sum_{v \in U} \delta_{v} \in \mathbb{C} V$ for every $U \subseteq V$. That is, $\delta_{U}(u)=1$ if $u \in U$ and 0 otherwise. In particular, $\delta_{V}$ is the constant function 1 on $V$ (all-one column vector). It is not difficult to verify the following result.

Lemma 5.1. ([9, Lemma 2(ii)]; see also [14, Lemma 9.3.2]) Let $\Gamma=(V, E)$ be a d-regular graph. A partition $\left\{V_{1}, \ldots, V_{m}\right\}$ of $V$ is equitable if and only if the subspace of $\mathbb{C} V$ spanned by $\delta_{V_{1}}, \ldots, \delta_{V_{m}}$ is invariant under the linear mapping $\varphi$.

In the sequel, for an equitable partition $\pi=\left\{V_{1}, \ldots, V_{m}\right\}$ we denote by $\mathbb{C} V_{\pi}$ the subspace of $\mathbb{C} V$ spanned by $\delta_{V_{1}}, \ldots, \delta_{V_{m}}$. Then $\operatorname{dim}\left(\mathbb{C} V_{\pi}\right)=m$. Denote by $\varphi_{\pi}$ the restriction of $\varphi$ to $\mathbb{C} V_{\pi}$. It is straightforward to verify that the matrix $A_{\pi}$ of $\varphi_{\pi}$ with respect to the 'standard' basis $\delta_{V_{1}}, \ldots, \delta_{V_{m}}$ of $\mathbb{C} V_{\pi}$ is given by

$$
A_{\pi}=\left(b_{i j}\right)_{1 \leq i, j \leq m}
$$

where as before $b_{i j}=\left|\Gamma(u) \cap V_{j}\right|$ for $u \in V_{i}$.

Lemma 5.2. Let $\Gamma=(V, E)$ be a d-regular graph. A subset $C \subseteq V$ is a total perfect code in $\Gamma$ if and only if

$$
\varphi\left(\delta_{C}\right)=\delta_{V} .
$$


Proof We have $\varphi\left(\delta_{C}\right)(w)=\sum_{v \in C} \varphi\left(\delta_{v}\right)(w)=\sum_{v \in C} a_{w v}=|\Gamma(w) \cap C|$ for $w \in V$. From this and the definition of a total perfect code the result follows immediately.

We now prove the following counterpart of [9, Proposition 3] by using a similar approach.

Theorem 5.3. Let $\Gamma=(V, E)$ be a d-regular graph and $C$ a total perfect code in $\Gamma$. Then for every equitable partition $\pi=\left\{V_{1}, \ldots, V_{m}\right\}$ of $\Gamma$ there exists a vector $\left(k_{1}, \ldots, k_{m}\right)^{T} \in \mathbb{Q}^{m}$ such that

$$
\begin{gathered}
\left|V_{i} \cap C\right|=\left(\frac{1}{d}+k_{i}\right)\left|V_{i}\right|, \quad i=1, \ldots, m \\
A_{\pi}\left(k_{1}, \ldots, k_{m}\right)^{T}=(0, \ldots, 0)^{T} .
\end{gathered}
$$

Proof Define

$$
p: \mathbb{C} V \rightarrow \mathbb{C} V_{\pi}, \quad f \mapsto p(f):=\sum_{i=1}^{m}\left(\frac{\sum_{v \in V_{i}} f(v)}{\left|V_{i}\right|}\right) \delta_{V_{i}} .
$$

Then $p$ is a linear mapping from $\mathbb{C} V$ to $\mathbb{C} V_{\pi}$. Denote by $P$ the matrix of $p$ with respect to the 'standard' basis $\left\{\delta_{v}: v \in V\right\}$. It is straightforward to verify that the $(u, v)$-entry of $P$ is given by $P_{u v}=\delta_{V_{u}}(v) /\left|V_{u}\right|$, where $V_{u}$ is the unique part $V_{i}$ of $\pi$ containing $u$. Using this and the assumption that $\pi$ is equitable, one can verify (see [9]) that $P A=A P$. In other words, $p \circ \varphi=\varphi \circ p$.

Since $C$ is a total perfect code, we have $\varphi\left(\delta_{C}\right)=\delta_{V}$ by (3). This together with $p \circ \varphi=\varphi \circ p$ implies $(\varphi \circ p)\left(\delta_{C}\right)=(p \circ \varphi)\left(\delta_{C}\right)=p\left(\delta_{V}\right)=\delta_{V}$. Since $\Gamma$ is $d$-regular, we have $\varphi\left(\frac{1}{d} \delta_{V}\right)=\delta_{V}$ and hence $(\varphi \circ p)\left(\frac{1}{d} \delta_{V}\right)=(p \circ \varphi)\left(\frac{1}{d} \delta_{V}\right)=p\left(\delta_{V}\right)=\delta_{V}$. By the definition of $p$, we have $p\left(\delta_{C}\right)-p\left(\frac{1}{d} \delta_{V}\right) \in \mathbb{C} V_{\pi}$. Therefore, $\varphi_{\pi}\left(p\left(\delta_{C}\right)-p\left(\frac{1}{d} \delta_{V}\right)\right)=\mathbf{0}$, that is, $p\left(\delta_{C}\right)-p\left(\frac{1}{d} \delta_{V}\right) \in$ $\operatorname{Ker}\left(\varphi_{\pi}\right)$. On the other hand, since $p\left(\delta_{C}\right)-p\left(\frac{1}{d} \delta_{V}\right) \in \mathbb{C} V_{\pi}$, there exists $\left(k_{1}, \ldots, k_{m}\right)^{T} \in \mathbb{C}^{m}$ such that $p\left(\delta_{C}\right)-p\left(\frac{1}{d} \delta_{V}\right)=\sum_{i=1}^{m} k_{i} \delta_{V_{i}}$. Since this vector is in $\operatorname{Ker}\left(\varphi_{\pi}\right)$ and $\varphi_{\pi}$ has matrix $A_{\pi}=\left(b_{i j}\right)_{1 \leq i, j \leq m}$ with respect to the basis $\delta_{V_{1}}, \ldots, \delta_{V_{m}}$, it follows that $\left(k_{1}, \ldots, k_{m}\right)^{T}$ satisfies (5). We have $p\left(\delta_{C}\right)=\sum_{i=1}^{m}\left(\frac{\left|V_{i} \cap C\right|}{\left|V_{i}\right|}\right) \delta_{V_{i}}$ and $p\left(\frac{1}{d} \delta_{V}\right)=\frac{1}{d} \delta_{V}=\frac{1}{d} \sum_{i=1}^{m} \delta_{V_{i}}$. Thus $\sum_{i=1}^{m}\left(\frac{\left|V_{i} \cap C\right|}{\left|V_{i}\right|}\right) \delta_{V_{i}}-$ $\frac{1}{d} \sum_{i=1}^{m} \delta_{V_{i}}=\sum_{i=1}^{m} k_{i} \delta_{V_{i}}$. Since $\delta_{V_{1}}, \ldots, \delta_{V_{m}}$ are independent, we have $\frac{\left|V_{i} \cap C\right|}{\left|V_{i}\right|}-\frac{1}{d}=k_{i}$ for each $i$. It is obvious that all coordinates $k_{i}$ are rationals.

Corollary 5.4. Let $\Gamma=(V, E)$ be a d-regular graph.

(a) If $\Gamma$ admits a total perfect code, then for any equitable partition $\pi=\left\{V_{1}, \ldots, V_{m}\right\}$ of $\Gamma$, either 0 is an eigenvalue of $A_{\pi}$ with an eigenvector $\left(k_{1}, \ldots, k_{m}\right)$ giving by (4), or d divides $\left|V_{i}\right|$ for each $i=1, \ldots, m$.

(b) If $\Gamma$ admits a total perfect code and $d \geq 2$, then 0 is an eigenvalue of $\Gamma$ (obtained from the trivial equitable partition $\{\{v\}: v \in V\})$ and $\left(k_{v}\right)_{v \in V}$ is a corresponding eigenvector, where $k_{v}=1-\frac{1}{d}$ if $v \in C$ and $k_{v}=-\frac{1}{d}$ if $v \notin C$.

Proof The truth of (a) follows from Theorem 5.3 immediately. Applying (a) to the trivial equitable partition $\{\{v\}: v \in V\}$, we obtain (b) by noting that $d \geq 2$ is not a divisor of 1 .

Part (b) in Corollary 5.4 is parallel to the well known result [14, Lemma 9.3.4] that a regular graph admitting a perfect 1-code should have -1 as an eigenvalue. 


\subsection{A necessary condition}

The purpose of this subsection is to establish the following analogy of [9, Theorem 6] by using a similar approach. Denote by $d_{\chi}$ the degree of a character $\chi$ of a group. As $\chi$ is a class function, for a conjugacy class $K$ we write $\chi(K)=\chi(x)$ where $x \in K$.

Theorem 5.5. Let $G$ be a group and $S$ a union of $s$ conjugacy classes of $G$ with $1 \notin S$ and $S^{-1}=S$. Let $\mathcal{Q}$ be the set of irreducible characters $\chi$ of $G$ such that $\sum_{K} \frac{|K| \chi(K)}{d_{\chi}}=0$, where $K$ runs over all conjugacy classes of $G$ contained in $S$. If $\operatorname{Cay}(G, S)$ admits a total perfect code, then

(a) $\sum_{\chi \in \mathcal{Q}} d_{\chi}^{2} \geq|S|-1$;

(b) $|\mathcal{Q}| \geq s$.

In the case when $G$ is abelian, it has exactly $|G|$ irreducible characters all of which are linear. Thus all $d_{\chi}=1$ and $\mathcal{Q}$ consists of those $\chi$ such that $\chi(S):=\sum_{g \in S} \chi(g)=0$. Since $G$ is abelian, we have $|S|=s$ and $\chi(S)$, with $\chi$ running over all irreducible characters of $G$, are precisely the eigenvalues of Cay $(G, S)$. Thus (a) and (b) above yield $|\mathcal{Q}| \geq s-1$ and $|\mathcal{Q}| \geq s$, respectively. Therefore, Theorem 5.5 implies:

Corollary 5.6. Let $G$ be an abelian group and $S$ a subset of $G$ with $1 \notin S$ and $S^{-1}=S$. Then $\operatorname{Cay}(G, S)$ admits a total perfect code only if the multiplicity of 0 as an eigenvalue of $\operatorname{Cay}(G, S)$ is at least $|S|$ (that is, $G$ has at least $|S|$ irreducible characters $\chi$ such that $\sum_{g \in S} \chi(g)=0$ ).

The rest of this subsection is devoted to the proof of Theorem 5.5. We assume that $G$ and $S$ are as in Theorem 5.5 and denote $\Gamma=\operatorname{Cay}(G, S)$. To be explicit we assume

$$
S=\cup_{i=1}^{s} K_{i},
$$

where each $K_{i}$ is a conjugacy class of $G$ with $K_{i}^{-1}$ also contained in $S$. Without loss of generality we may assume that the set of all conjugacy classes of $G$ is

$$
\pi=\left\{K_{1}, \ldots, K_{s}, K_{s+1}, \ldots, K_{m}\right\} .
$$

Then $\mathbb{C} G_{\pi}$ is the vector space of class functions of $G$. Since $S$ is closed under conjugation, the inner automorphism group $\operatorname{Inn}(G)$ of $G$ is a subgroup of $\operatorname{Aut}(\Gamma)$ with respect to its natural action on $G$. Since the orbits of $\operatorname{Inn}(G)$ on $G$ are precisely the conjugacy classes of $G$, it follows that $\pi$ is an equitable partition of $\Gamma$. We will apply Theorem 5.3 to this particular partition in the proof of Theorem 5.5 .

Let

$$
\lambda_{G}: G \rightarrow \operatorname{Sym}(\mathbb{C} G), \quad g \mapsto \lambda_{G}(g)
$$

be the left regular permutation representation of $G$, defined by

$$
\left(\lambda_{G}(g) f\right)(x)=f\left(g^{-1} x\right), \quad f \in \mathbb{C} G, x \in G .
$$

It can be verified that

$$
\lambda_{G}(g) \delta_{v}=\delta_{g v}, g, v \in G .
$$

Let $\varphi: \mathbb{C} G \rightarrow \mathbb{C} G$ be the adjacency linear mapping as in $(2)$ for $V=G$ and $\Gamma=\operatorname{Cay}(G, S)$.

Lemma 5.7. $\varphi=\sum_{g \in S} \lambda_{G}(g)$. 
Proof We have $\left(\sum_{g \in S} \lambda_{G}(g)\right)\left(\delta_{v}\right)=\sum_{g \in S} \lambda_{G}(g)\left(\delta_{v}\right)=\sum_{g \in S} \delta_{g v}=\sum_{u \in \Gamma(v)} \delta_{u}=\varphi\left(\delta_{v}\right)$ for any $v \in G$. Since $\left\{\delta_{v}: v \in G\right\}$ is a basis of $\mathbb{C} G$, the result follows.

We remark that Lemma 5.7 holds for any $S \subset G$ that is not necessarily a union of conjugacy classes of $G$.

Lemma 5.8. ([9, Lemma 5]) The irreducible characters of $G$ (i) constitute a basis of $\mathbb{C} G_{\pi}$, and (ii) are eigenvectors of $\varphi_{\pi}$. More explicitly, for any irreducible character $\chi$ of $G$,

$$
\varphi_{\pi}(\chi)=\left(\sum_{i=1}^{s} \frac{\left|K_{i}\right| \chi\left(K_{i}\right)}{d_{\chi}}\right) \chi .
$$

The truth of (i) is a well known result in group theory as $\pi$ is the partition of $G$ into conjugacy classes. Part (ii) was proved in [9] with the help of [11, (5.4)].

Proof of Theorem 5.5 Suppose that $C$ is a total perfect code in $\Gamma$. By Lemma 3.1(a), we may assume $1 \in C$ without loss of generality. Since $S$ is closed under conjugation, by Lemma 3.1(c), $\{C g: g \in S\}$ is a partition of $G$, and hence $\delta_{C g}, g \in S$ are independent vectors of $\mathbb{C} G$. On the other hand, by Lemma 3.1(a) each $C g$ with $g \in S$ is a total perfect code of $\Gamma$. Thus by Lemma 5.2, $f=\delta_{C g}, g \in S$ are solutions to the linear equation $\varphi(f)=\delta_{G}$. Since these are $|S|$ independent solutions, the homogeneous equation $\varphi(f)=0$ has at least $|S|-1$ independent solutions. In other words,

$$
\operatorname{dim}(\operatorname{Ker}(\varphi)) \geq|S|-1 .
$$

We now compute $\operatorname{dim}(\operatorname{Ker}(\varphi))$ by way of the irreducible characters of $G$. Let $\chi_{1}, \ldots, \chi_{m}$ be such characters (see Lemma 5.8(i)). It is well known that in the decomposition of the regular representation into a direct sum of irreducible representations, the number of each irreducible representation is equal to its degree. Thus, by Lemma 5.7 and (6), we obtain

$$
\varphi=\bigoplus_{W}\left(\sum_{i=1}^{s} \frac{\left|K_{i}\right|}{d_{\chi_{W}}} \chi_{W}\left(K_{i}\right)\right) \operatorname{Id}_{W},
$$

where the direct sum runs over all irreducible representations $\left(\rho_{W}, W\right)$ in the decomposition of $\lambda_{G}$ (into a direct sum of irreducible representations), and $\mathrm{Id}_{W}$ is the identity mapping from $W$ to itself. Therefore, $\operatorname{dim}(\operatorname{Ker}(\varphi))=\sum_{\chi \in \mathcal{Q}} d_{\chi}^{2}$. This together with (7) yields $\sum_{\chi \in \mathcal{Q}} d_{\chi}^{2} \geq|S|-1$ as claimed in (a).

It remains to prove (b). We may assume $K_{s+1}=\{1\}$ without loss of generality. Choose an arbitrary element $g_{j} \in K_{j}, 1 \leq j \leq s+1$. (Note that $g_{s+1}=1$.) By Lemma 3.1(a), each $C g_{j}$ is a total perfect code in $\Gamma$.

We now prove that the rank of the matrix $\left(\left|K_{i} \cap C g_{j}\right|\right)_{1 \leq i \leq m, 1 \leq j \leq s+1}$ is equal to $s+1$. We show first that

$$
\left|K_{i} \cap C g_{j}\right|= \begin{cases}1, & \text { if } 1 \leq i=j \leq s \\ 0, & \text { if } 1 \leq i \neq j \leq s .\end{cases}
$$

Suppose that $K_{i} \cap C g_{j} \neq \emptyset$, where $1 \leq i, j \leq s$. Then there exist $x \in K_{i}$ and $y \in C$ such that $x=y g_{j}$. As $K_{i} \subseteq S$ and $1 \notin S$, we have $1 \neq x \in S$. Moreover, since $S$ is closed under conjugation and $g_{j} \in S$, we have $x y^{-1}=y g_{j} y^{-1} \in S$. Hence $x$ is adjacent to 1 and $y$ in $\Gamma$. Since $1, y \in C$ and $C$ is a total perfect code, it follows that $y=1$. In particular, $x=g_{j} \in K_{i} \cap K_{j}$. Thus $i=j$ and moreover the only possible common element of $K_{i}$ and $C g_{i}$ is $g_{i}$. On the other hand, since $1 \in C$, we do have $g_{i} \in K_{i} \cap C g_{i}$. Therefore, $K_{i} \cap C g_{i}=\left\{g_{i}\right\}$ and (9) is proved.

Now suppose $1 \leq i \leq s+1$ and $j=s+1$, and consider $\left|K_{i} \cap C g_{s+1}\right|=\left|K_{i} \cap C\right|\left(\right.$ as $\left.g_{s+1}=1\right)$. As $K_{s+1}=\{1\}$ and $1 \in C$, we have $\left|K_{s+1} \cap C g_{s+1}\right|=1$. Since $\Gamma(1)=S$ and $1 \in C$ is adjacent to 
exactly one vertex in $C$, we have $|S \cap C|=1$. This together with $S=\cup_{i=1}^{s} K_{i}$ implies that there exists a unique $i^{*}$ with $1 \leq i^{*} \leq s$ such that $\left|K_{i^{*}} \cap C\right|=1$ and $\left|K_{i} \cap C\right|=0$ for $1 \leq i \leq s$ with $i \neq i^{*}$. Combining this with (9), we obtain that the first $s+1$ rows of $\left(\left|K_{i} \cap C g_{j}\right|\right)_{1 \leq i \leq m, 1 \leq j \leq s+1}$ form the following submatrix:

$$
\left[\begin{array}{cc}
I_{s} & e_{i^{*}}^{T} \\
0 & 1
\end{array}\right]
$$

where $I_{s}$ is the $s \times s$ identity matrix and $e_{i^{*}}=(0, \ldots, 0,1,0, \ldots, 0)$ with 1 in the $i^{*}$ th coordinate. In particular, the rank of $\left(\left|K_{i} \cap C g_{j}\right|\right)_{1 \leq i \leq m, 1 \leq j \leq s+1}$ is equal to $s+1$ as claimed.

By (6), $f \in \operatorname{Ker}\left(\varphi_{\pi}\right) \Leftrightarrow f=\sum_{\chi \in \mathcal{Q}} a_{\chi} \chi$, for some $a_{\chi} \in \mathbb{C} \Leftrightarrow f=\sum_{\chi \in \mathcal{Q}} a_{\chi}\left(\sum_{i=1}^{m} \chi\left(K_{i}\right) \delta_{K_{i}}\right)$ $\Leftrightarrow f=\sum_{i=1}^{m}\left(\sum_{\chi \in \mathcal{Q}} a_{\chi} \chi\left(K_{i}\right)\right) \delta_{K_{i}}$. Note that $a_{\chi}$ does not rely on $i$.

Since each $C g_{j}$ is a total perfect code in $\Gamma$, by Theorem 5.3 and the computation above, for $1 \leq i \leq m$ and $1 \leq j \leq s+1$, there exist $a_{\chi, j} \in \mathbb{C}$ and $\chi \in \mathcal{Q}$ such that

$$
\left|K_{i} \cap C g_{j}\right|=\frac{\left|K_{i}\right|}{d}+\left(\sum_{\chi \in \mathcal{Q}} a_{\chi, j} \chi\left(K_{i}\right)\right)\left|K_{i}\right|
$$

In other words,

$\left(\left|K_{i} \cap C g_{j}\right|\right)_{1 \leq i \leq m, 1 \leq j \leq s+1}=\frac{1}{d}\left[\begin{array}{ccc}\left|K_{1}\right| & \cdots & \left|K_{1}\right| \\ \vdots & & \vdots \\ \left|K_{m}\right| & \cdots & \left|K_{m}\right|\end{array}\right]+\left(\left|K_{i}\right| \chi\left(K_{i}\right)\right)_{1 \leq i \leq m, \chi \in \mathcal{Q}} \cdot\left(a_{\chi, j}\right)_{\chi \in \mathcal{Q}, 1 \leq j \leq s+1}$.

Since as shown above the matrix on the left-hand side has rank $s+1$ and the first term on the right-hand side has rank one, the rank of the product $\left(\left|K_{i}\right| \chi\left(K_{i}\right)\right) \cdot\left(a_{\chi, j}\right)$ is at least $s$. Thus the rank of $\left(\left|K_{i}\right| \chi\left(K_{i}\right)\right)_{1 \leq i \leq m, \chi \in \mathcal{Q}}$ is at least $s$. Consequently, $|\mathcal{Q}| \geq s$ as required in (b).

Example 5.9. The characters of a cyclic group $C_{n}=\langle a\rangle$ of order $n$ are $\chi_{k}, 0 \leq k \leq n-1$, defined by $\chi_{k}\left(a^{j}\right)=\omega^{k j}$, where $\omega=e^{2 \pi i / n}$. By Corollary 5.6, a circulant $\operatorname{Cay}\left(C_{n}, S\right)$ (where $\left.S^{-1}=S \subseteq C_{n} \backslash\{1\}\right)$ admits a total perfect code only if there are at least $|S|$ integers $k$ between 0 and $n-1$ such that $\sum_{j: a^{j} \in S} \omega^{k j}=0$.

\subsection{Another necessary condition}

Let $\Gamma=\operatorname{Cay}(G, S)$ be the Cayley graph in Theorem 5.5 and $H$ a subgroup of $G$. Denote by

$$
\pi(H)=\left\{x_{1} H, \ldots, x_{m} H\right\}
$$

the partition of $G$ into left cosets of $H$ in $G$, where $m=|G: H|$ and $x_{1}, \ldots, x_{m}$ are a set of representatives of such cosets. Since these cosets are the $H$-orbits under the action of $H$ on $G$ by right multiplication, and $H$ can be viewed as a subgroup of $\operatorname{Aut}(\Gamma)$, it follows that $\pi(H)$ is an equitable partition of $\Gamma$. The 'standard' basis of $\mathbb{C} G_{\pi(H)}$ is $\left\{\delta_{x_{1} H}, \ldots, \delta_{x_{m} H}\right\}$. Let $\lambda_{G}^{H}: G \rightarrow$ $\operatorname{Sym}\left(\mathbb{C} G_{\pi(H)}\right)$ be the representation of $G$ with degree $|G: H|$ defined by $\lambda_{G}^{H}(g)\left(\delta_{x_{i} H}\right)=\delta_{g x_{i} H}$ for $g \in G$ and $1 \leq i \leq m$. The following result is analogous to [9, Theorem 7].

Theorem 5.10. Let $G$ be a group and $S$ a union of conjugacy classes of $G$ with $1 \notin S$ and $S^{-1}=S$. Suppose $H$ is a subgroup of $G$ such that $\sum_{K} \frac{|K| \chi(K)}{d_{\chi}} \neq 0$ for every irreducible character $\chi$ of $G$ occurring in the decomposition of $\lambda_{G}^{H}$ (into a direct sum of irreducible characters), where $K$ runs over all conjugacy classes contained in $S$. Then every total perfect code in $\operatorname{Cay}(G, S)$ intersects every left coset of $H$ in $G$ at exactly $|H| /|S|$ elements. In particular, if $|S|$ does not divide $|H|$, then $\operatorname{Cay}(G, S)$ has no total perfect code. 
Proof As in $\$ 5.2$, let $S=\cup_{i=1}^{s} K_{i}$ and let $\varphi: \mathbb{C} G \rightarrow \mathbb{C} G$ be the linear mapping defined in (2) for $V=G$ and $\Gamma=\operatorname{Cay}(G, S)$. Similar to Lemma 5.7, a straightforward computation yields [9]

$$
\varphi_{\pi(H)}=\sum_{g \in S} \lambda_{G}^{H}(g)
$$

(That is, the matrix of $\varphi_{\pi(H)}$ is given by $A_{\pi(H)}=\sum_{g \in S} \lambda_{G}^{H}(g)$ when $\lambda_{G}^{H}(g)$ is interpreted as the permutation matrix of the permutation $\delta_{x_{i} H} \mapsto \delta_{g x_{i} H}, i=1, \ldots, m$ of the basis $\left\{\delta_{x_{1} H}, \ldots, \delta_{x_{m} H}\right\}$ of $\left.\mathbb{C} G_{\pi(H)}.\right)$ Based on this and similar to $(8)$, one can verify that

$$
\varphi_{\pi(H)}=\bigoplus_{W}\left(\sum_{i=1}^{s} \frac{\left|K_{i}\right|}{d_{\chi_{W}}} \chi_{W}\left(K_{i}\right)\right) \operatorname{Id}_{W},
$$

where the direct sum runs over all irreducible representations $\left(\rho_{W}, W\right)$ in the decomposition of $\lambda_{G}^{H}$ (into a direct sum of irreducible representations). Thus $\operatorname{det}\left(A_{\pi(H)}\right) \neq 0$ if and only if $\sum_{i=1}^{s} \frac{\left|K_{i}\right|}{d_{\chi_{W}}} \chi_{W}\left(K_{i}\right) \neq 0$ for every $W$ in 10 . In this case, by $44-(5)$ applied to $\pi(H)$, for any total perfect code $C$ in $\Gamma$ we have $\left|x_{i} H \cap C\right|=|H| /|S|$, which occurs only when $|S|$ is a divisor of $|H|$.

Acknowledgements The author is grateful to three anonymous referees for their helpful comments which led to improvement of Theorem 5.5(b) and betterment of presentation of the paper. This research was supported by the Australian Research Council (FT110100629).

\section{References}

[1] R. Ahlswede, H. K. Aydinian and L. H. Khachatrian, On perfect codes and related concepts, Designs, Codes and Cryptography 22 (2001), 221-237.

[2] C. Araujo and I. Dejter, Lattice-like total perfect codes, Discuss. Math. Graph Theory 34 (2014), no. $1,57-74$.

[3] E. Bannai, On perfect codes in the Hamming schemes $H(n, q)$ with $q$ arbitrary, J. Combin. Theory Ser. A 23 (1977), no. 1, 52-67.

[4] N. L. Biggs, Perfect codes in graphs, J. Combin. Theory Ser. B 15 (1973), 289-296.

[5] R. Cowen, S. H. Hechler, J. W. Kennedy and A. Steinberg, Odd neighborhood transversals on grid graphs, Discrete Math. 307 (2007), 2200-2208.

[6] I. J. Dejter, Perfect domination in regular grid graphs, Australas. J. Combin. 42 (2008), 99-114.

[7] I. J. Dejter and O. Serra, Efficient dominating sets in Cayley graphs, Discrete Appl. Math. 129 (2003) 319-328.

[8] P. Delsarte, An algebraic approach to the association schemes of coding theory, Philips Res. Rep. Suppl. 10, 1973.

[9] G. Etienne, Perfect codes and regular partitions in graphs and groups, European J. Combin. 8 (1987), no. $2,139-144$.

[10] T. Etzion, Product constructions for perfect Lee codes, IEEE Trans. Inform. Theory $5 \mathbf{7}$ (2011), no. $11,7473-7481$.

[11] W. Feit, Characters of Finite Groups, Benjamin, 1967.

[12] H. Gavlas, K. Schultz and P. Slater, Efficient open domination in graphs, Sci. Ser. A Math. Sci. (N.S.) $6(1994 / 00)(2003), 77-84$.

[13] A-A. Ghidewon, R. H. Hammack and D. T. Taylor, Total perfect codes in tensor products of graphs, Ars Combin. 88 (2008), 129-134. 
[14] C. Godsil and G. Royle, Algebraic Graph Theory, Springer-Verlag, New York, 2001.

[15] I. Gorodezky, Dominating Sets in Kneser Graphs, Master Thesis, The University of Waterloo, 2007.

[16] P. Hammond and D. H. Smith, Perfect codes in the graphs $O_{k}$, J. Combin. Theory Ser. B 19, no. 3, 239-255.

[17] T. W. Haynes, S. T. Hedetniemi and P. Slater, Fundamentals of Domination in Graphs, Marcel Dekker, Inc., New York, 1998.

[18] O. Heden, Perfect codes in antipodal distance-transitive graphs, Math. Scand. 35 (1974), 29-37.

[19] O. Heden, A survey of perfect codes, Adv. Math. Commun. 2 (2008), no. 2, 223-247.

[20] W. F. Klostermeyer and J. L. Goldwasser, Total perfect codes in grid graphs, Bull. Inst. Combin. Appl. 46 (2006), 61-68.

[21] S. Klavzăr, U. Milutinović and C. Petr, 1-perfect codes in Sierpiński graphs, Bull. Austral. Math. Soc. 66 (2002), no. 3, 369-384.

[22] J. Kratochvíl, Perfect codes over graphs, J. Combin. Theory Ser. B 40 (1986), no. 2, 224-228.

[23] M. Knor and P. Potočnik, Efficient domination in cubic vertex-transitive graphs, European J. Combin. 33 (2012), no. 8, 1755-1764.

[24] D. Kuziak, I. Peterin and I. G. Yero, Efficient open domination in graph products, Discrete Math. and Theoret. Comp. Sci. 16 (2014), no. 1, 105-120.

[25] J. Lee, Independent perfect domination sets in Cayley graphs, J. Graph Theory 37 (2001), 213-219.

[26] C-K. Li and I. Nelson, Perfect codes on the towers of Hanoi graph, Bull. Austral. Math. Soc. 57 (1998), no. 3, 367-376.

[27] M. Livingston and Q. F. Stout, Perfect dominating sets, Congr. Numer. 79 (1990), 187-203.

[28] C. Martínez, R. Beivide and E. Gabidulin, Perfect codes for metrics induced by circulant graphs, IEEE Trans. Inform. Theory 53 (2007), no. 9, 3042-3052.

[29] C. Martínez, R. Beivide and E. Gabidulin, Perfect codes from Cayley graphs over Lipschitz integers, IEEE Trans. Inform. Theory 55 (2009), no. 8, 3552-3562.

[30] M. Mollard, On perfect codes in Cartesian products of graphs, European J. Combin. 32 (2011), no. 3, 398-403.

[31] N. Obradović, J. Peters and G. Ružić, Efficient domination in circulant graphs with two chord lengths, Inform. Process. Lett. 102 (2007), no. 6, 253-258.

[32] C. Payan, On the chromatic number of cube-like graphs, Discrete Math. 103 (1992), no. 3, 271-277.

[33] K. Reji Kumar and G. MacGillivray, Efficient domination in circulant graphs, Discrete Math. 313 (2013), no. 6, 767-771.

[34] J. J. Rotman, An Introduction to the Theory of Groups, 4th ed., Springer-Verlag, New York, 1995.

[35] S. Špacapan, Perfect codes in direct products of cycles, Electronic Notes in Discrete Math. 22 (2005), 201-205.

[36] D. H. Smith, An improved version of Lloyd's theorem, Discrete Math. 15 (1976), no. 2, $175-184$.

[37] D. H. Smith, Perfect codes in the graphs $O_{k}$ and $L\left(O_{k}\right)$, Glasgow Math. J. 21 (1980), no. 2, $169-172$.

[38] M. Schwartz and T. Etzion, Codes and anticodes in the Grassman graph, J. Combin. Theory Ser. A 97 (2002), 27-42.

[39] S. Terada, Perfect codes in SL(2,2f), European J. Combin. 25 (2004), no. 7, 1077-1085.

[40] J. H. van Lint, A survey of perfect codes, Rocky Mountain J. Math. 5 (1975), 199-224.

[41] J. Žerovnik, Perfect codes in direct products of cycles-a complete characterization, Adv. in Appl. Math. 41 (2008), no. 2, 197-205.

[42] S. Zhou, Cyclotomic graphs and perfect codes, preprint, http://arxiv.org/abs/1502.03272. 